Doug Geisler, Eva K. Grebel, and Dante Minniti, eds.

\title{
Age as the Second Parameter in NGC 288/NGC 362?
}

\author{
M. Catelan \\ Hubble Fellow, University of Virginia, Department of Astronomy, \\ P.O. Box 3818, Charlottesville, VA 22903-0818, USA \\ M. Bellazzini, F. R. Ferraro, F. Fusi Pecci, S. Galleti \\ Osservatorio Astr. di Bologna, via Ranzani 1, I-40126 Bologna, Italy
}

W. B. Landsman

Raytheon ITSS, NASA GSFC, Greenbelt, MD 20771, USA

\begin{abstract}
We derive the age difference between NGC 288 and NGC 362 using the bimodal horizontal branch (HB) of NGC 1851 as a "bridge" in the CMD analysis. Our results suggest that NGC 288 is older than both NGC 362 and NGC 1851 by $\simeq 2 \pm 1$ Gyr. A new grid of synthetic HBs discloses that age may be the second parameter (2ndP) for this pair provided that the Carretta \& Gratton (1997) metallicity scale is to be preferred over the Zinn \& West (1984) scale. In addition, the dispersion in ZAHB mass is found to be about twice as large in NGC 362 as in NGC 288, probably due to the much higher central density of the former.
\end{abstract}

\section{Turnoff Ages: A Purely Differential Comparison}

Observations of NGC 288, NGC 362 and NGC 1851 were carried out during the same run at the $2.2 \mathrm{~m}$ ESO/MPI telescope (La Silla) with the EFOSC2 camera. The data were reduced and calibrated in an entirely homogeneous and selfconsistent way. The resulting $V, I$ CMDs allowed an application of the Stetson, VandenBerg, \& Bolte (1996) and VandenBerg (2000) "bridge test," as shown in Fig. 1. Assuming the same physics applies to the HB stars in all three clusters, NGC 288 is older than both NGC 362 and NGC 1851 by about $2 \pm 1$ Gyr.

\section{The Horizontal Branch Revisited}

We computed extensive grids of synthetic HBs to investigate anew whether such an age difference may be consistent with the hypothesis that age is the 2ndP for this pair. As shown in Fig. 2, we find that a higher metallicity, as in the Carretta \& Gratton (1997) scale, is more favorable to this hypothesis than the lower metallicity implied by the Zinn \& West (1984) scale (which had been adopted in all theoretical studies of the 2 ndP problem so far). In addition, our models show that the ZAHB mass dispersion in NGC 362 is about twice as large as in NGC 288, probably due to NGC 362's much higher central density. 

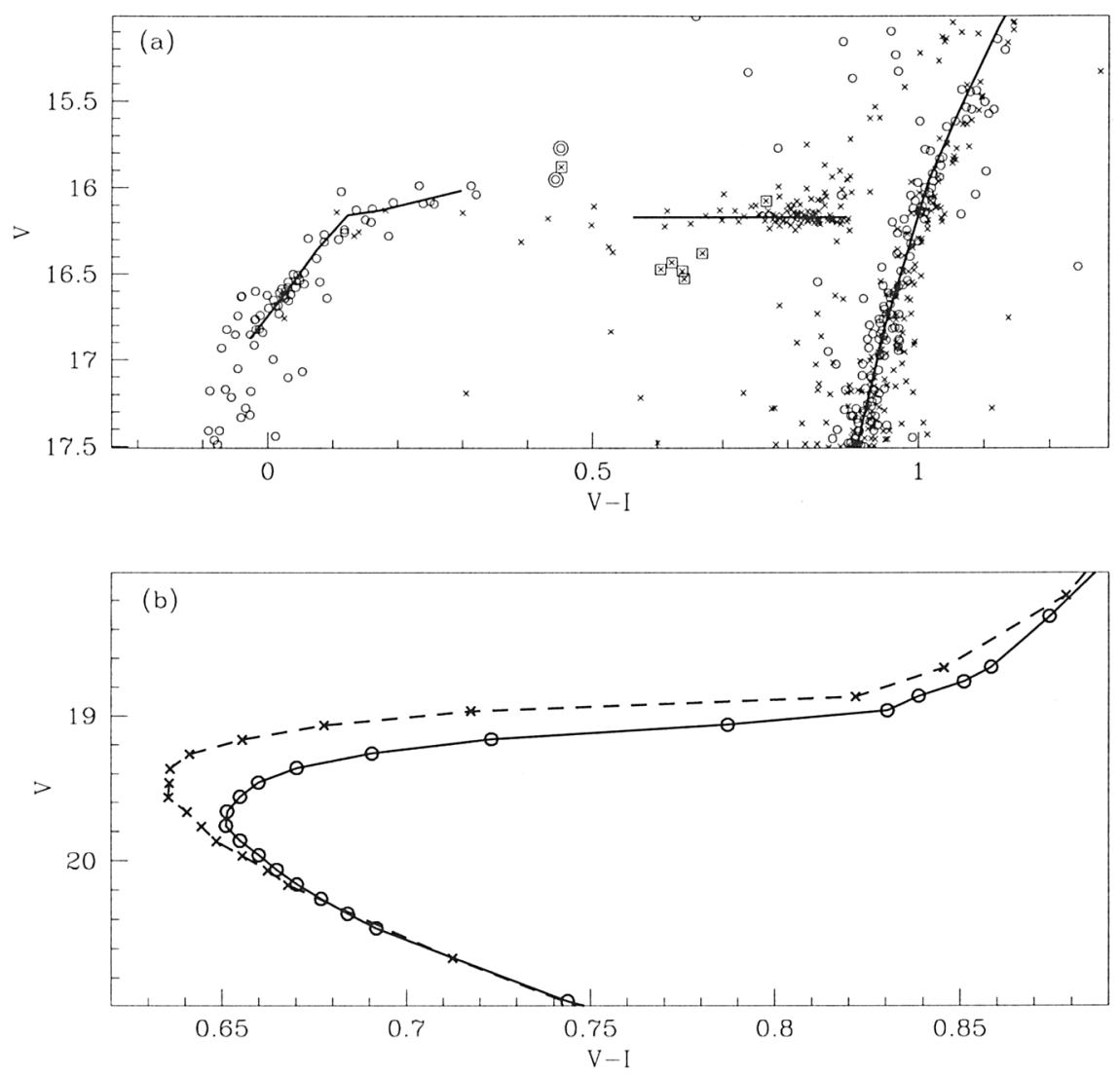

Figure 1. Our application of the "bridge method" to the clusters NGC 288 (open circles), NGC 362 (crosses) and NGC 1851 (the "bridge": lines, upper panel only). The upper panel shows the match of the HBs, whereas the lower panel depicts the resulting differences among the ridgelines around the turnoff region.

Acknowledgments. Financial support for M.C. was provided by NASA through Hubble Fellowship grant HF-01105.01-98A awarded by the STScI, which is operated by AURA for NASA under contract NAS5-26555.

\section{References}

Carretta, E., \& Gratton, R. 1997, A\&AS, 121, 95

Catelan, M. 2000, ApJ, 531, 826

Stetson, P. B., VandenBerg, D. A., \& Bolte, M. 1996, PASP, 108, 560

VandenBerg, D. A. 2000, ApJS, 129, 315

Zinn, R., \& West, M. J. 1984, ApJS, 55, 45 


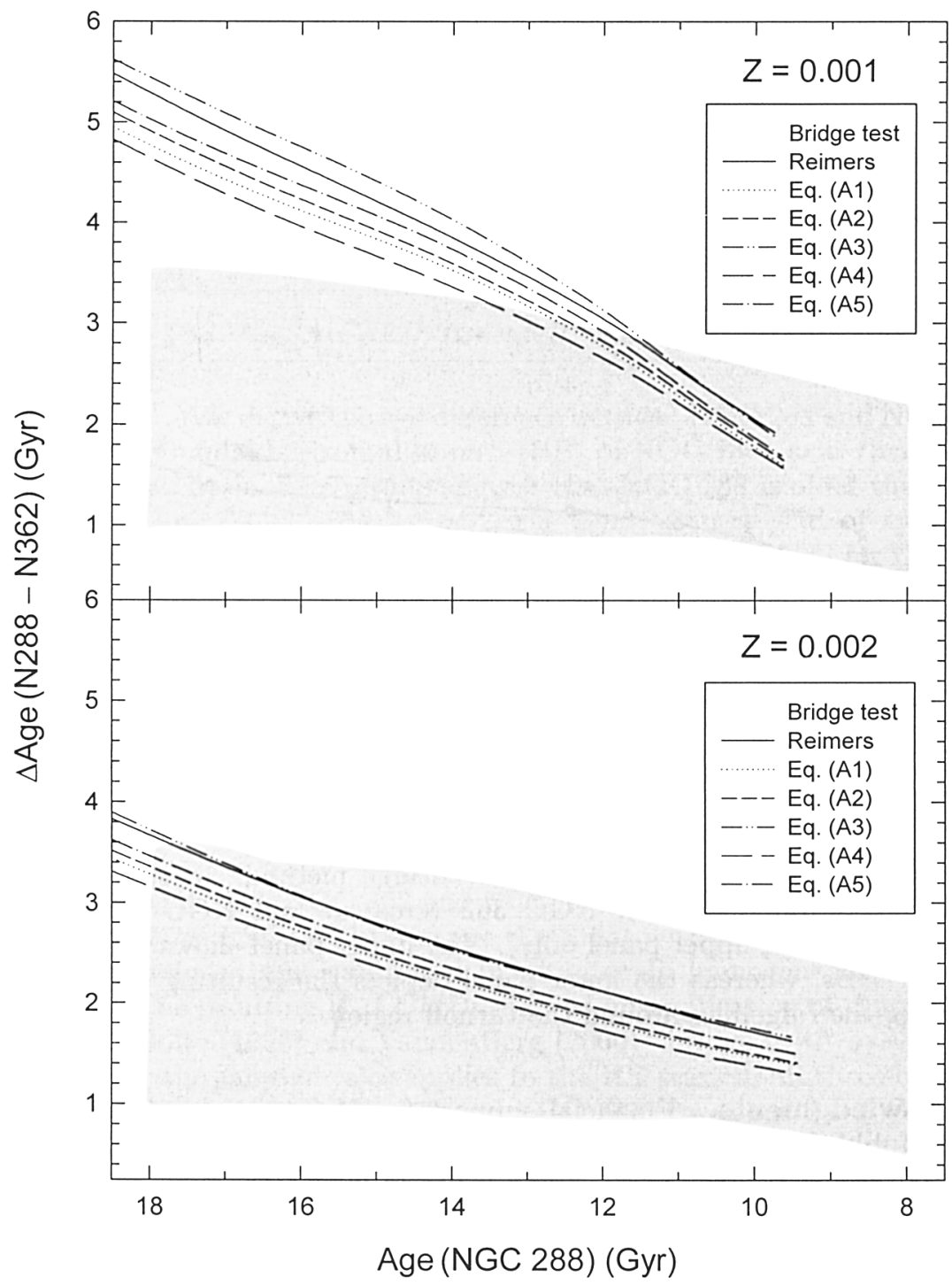

Figure 2. Age difference between NGC 288 and NGC 362, as predicted by theoretical models of HB morphology for several different assumptions about the mass loss on the RGB (lines; cf. Catelan 2000), compared against the result of the "bridge test" (gray bands). The upper panel is for a low, "Zinn \& West-like" metallicity, whereas the lower panel represents a higher, "Carretta \& Gratton-like" metallicity. 